Bangladesh J. Plant Taxon. 26(2): 231-247, 2019 (December)

(C) 2019 Bangladesh Association of Plant Taxonomists

\title{
ETHNOMEDICINAL STUDY FOCUSING ON ANTI-DIABETIC PLANTS USED BY THE COMMUNITY LIVING IN AND AROUND DHAKA
}

\author{
Mohammad Zashim Uddin ${ }^{1}$, Farhana Yesmin Mitu, Atiya Begum Rifat \\ and ABDULlah-Al-KaIUM \\ Department of Botany, University of Dhaka, Dhaka-1000, Bangladesh
}

Key words: Ethnomedicinal study; Anti-diabetic plants; Community; Conservation; Dhaka.

\begin{abstract}
The present article mainly focused on the ethnomedicinal plants used by the community living in and around Dhaka to manage the diabetes. Ethnomedicinal data were collected using semi-structured interviews with key informants during June 2017 to July 2018 followed by field interviews, plant interviews, checklist interviews and group discussion techniques. A total of 92 ethnomedicinal plant species under 46 families have been documented. These species were used to treat 55 ailments through 200 formularies. Herbs are the most common medicinal plants in the study area followed by trees, shrubs and climbers. Leaf is mostly used for the preparation of herbal medicine. Among the total 92 ethnomedicinal plants, 11 species have been used for diabetes management by the community people. Disease category diabetes showed maximum factor informant consensus value. Most cited ethnomedicinal plant species for the diabetes management are Gynura nepalensis DC., Coccinia grandis L. Voigt, Aloe vera (L.) Burm. f., Syzygium cumini (L.) Skeels, Swietenia mahagoni (L.) Jacq., Momordica dioica Roxb. ex. Willd., Catharanthus roseus (L.) G. Don, Streblus asper Lour., Bryophyllum pinnatum (Lamk.) Oken, Tamarindus indica L. and Scoparia dulcis L. The results in the present study for diabetes management were very preliminary and based on which sound conclusion was not possible. Further ethnopharmacological study is very essential on such species to validate their efficacy in the management of diabetes. Our findings also provide baseline data to establish a connection between the traditional users of medicinal plants and scientific communities, which can be substantial in novel drug discovery. Furthermore, ethnomedicinal data is of significant value for conservation managers and policy makers for sustainable management of ethnomedicinal plant species, which are under threat due to rapid urbanization.
\end{abstract}

\section{Introduction}

Diabetes is an increasingly serious health disorder to the mankind. It is related to other life killing diseases. The management of diabetes is a global problem until now and successful treatment is not yet discovered. Searching for new antidiabetic drugs from natural plants is still attractive because they contain substances which take alternative and safe effect on diabetes. Dhaka is the dwelling place for more than 20 million people. Due to inappropriate lifestyle, maximum people here in Bangladesh have been suffering from diabetes (Akter et al. 2014). All diabetic patients have not got access to modern medicines because of high cost and less availability. Poor sections of diabetic patients are bound to look for medicinal plants as an alternative source of medicine. They even get medicinal plants from vendors selling in the footpaths of Dhaka city. Unfortunately, knowledge on medicinal plants in and around Dhaka city is hard to be found in written form. However, some knowledge has been transferred orally from

${ }^{1}$ Corresponding author: zashim01@gmail.com 
one generation to another. Habitats of medicinal plants in and around Dhaka city is in a fragile state because of rapid urbanization, globalization, introduction of modern culture and anthropogenic pressures. Maximum ethnomedicinal plants will be lost before proper documentation and scientific evaluations for the welfare of mankind. Research on ethnomedicinal plants used for the treatment of diabetes has been carried out in the Indian subcontinent, by the Native Americans, Chinese, South Americans and Asian Indians (Mentreddy et al., 2005, Grover et al. 2002, Mukharjee et al. 2006). Recently, there has been a growing interest in the herbal medicine in care and management of diabetes both in developing and developed countries, due to their natural origin and less side effects (Modak et al. 2007, Hasani-Ranjbar et al. 2009).

In Bangladesh a good number of ethnobotanical works have been done in different upazilas, districts, and communities. Most noteworthy works are Mia and Huq (1988), Hassan and Khan (1986, 1996), Alam et al. (1996), Uddin et al. (2001), Khan et al. (2002), Yusuf et al. (2002), Uddin et al. (2004), Uddin et al. (2006), Yusuf (2006), Yusuf and Uddin (2006), Uddin and Roy (2007), Roy et al. (2008), Emily et al.(2010), Uddin et al. (2012), Sajib and Uddin, (2013), Haque et al. (2014) and Uddin and Hassan (2014), Uddin et al. (2015a), Uddin et al.(2015b), Uddin at al. (2017), Haque et al.(2017), Uddin and Haque (2018), Shethi and Uddin (2018), Uddin et al. (2019). No such works have covered ethnobotanical study focusing antidiabetic plants in and around Dhaka city. In order to save the knowledge of medicinal plants for the future drug research for diabetes management, an attempt has been made to record ethnomedicinal plants used by community living in and around Dhaka, to determine most cited medicinal plants for diabetes management and threats of medicinal plants with suggesting their conservation.

\section{Materials and Methods}

Dhaka district is located in between $23^{\circ} 53^{\prime}$ and $24^{\circ} 06^{\prime} \mathrm{N}$ and in between $90^{\circ} 01^{\prime}$ and $90^{\circ} 37^{\prime} \mathrm{E}$. The total area of the district is $1,464 \mathrm{~km}^{2}$. It is bounded by Gazipur and Tangail district to the north, Munshiganj district to the south, Narayanganj district to the east, Manikganj and Rajbari to the west. Dhaka is the capital city of Bangladesh and mainly stands on the bank of Buriganga river. Turag, Shitalakhya and Balu rivers encircle the city. It has been estimated that Dhaka city had 20 million people in 2018 and is one of the largest cities of the world. The area enjoys hot, wet and humid tropical climate. The public health and quality life in Dhaka city are severely affected by traffic congestion and air pollution. Water bodies and wetlands around Dhaka city are facing destruction as these are being filled up to construct multistoried buildings and other real estate developments. Dhaka has no natural forest cover but a good number of exotic tree species are planted in and around Dhaka city including parks, gardens, road dividers, footpaths and house yard. Luxuriant growth of seasonal herbs, aquatics and climbers were observed in and around Dhaka city during growing season. Some native tree species were also found in Dhaka. A good number of people possess traditional botanical knowledge and they use such plant species in their primary health care management.

The selected spots including Kamrangirchar, Keraniganj, Atibazar, Ashulia, Mirpur, Gulishtan, Sher-e-bangla nagar, Purbachal, Uttara, Boldagarden, Ramna park and DU campus area were visited during different seasons of 2017 and 2018. The data have been recorded through semistructured interviews, key informant discussions and informal conversations with community people and herbal practitioners (Alexiades 1996). During the field survey, information on uses of plants to treat humans, parts used, modes of preparation and administration have been documented along with vernacular names. A total of 162 people have been interviewed for the study age ranged between 20 to 80 . Among the informants majority are Muslims and the rest are from other 
religions. Education level is up to SSC and professionally they are farmers, day laborers, house wives, herbal practioners and small shopkeepers.

Voucher specimen for each medicinal plant has been collected and processed using standard herbarium techniques (Hyland 1972, Alexiades 1996). The specimens have been identified consulting different Floras viz., Hooker 1872-1897; Prain 1903; Uddin and Hassan 2004; Siddiqui et al. 2007 and Ahmed et al. 2008a, 2008b, 2009a, 2009b, 2009c, 2009d. Specimens available at Dhaka University Salar Khan Herbarium (DUSH) and Bangladesh National Herbarium have also been consulted in identifying the collected plant specimens. Voucher specimens have been deposited at DUSH. In order to estimate use diversity of the medicinal plants and to determine which plants are particularly interesting in the search for bioactive compounds, Factor of informant consensus (Fic) was calculated (Heinrich et al. 1998). Citation frequency (Cf) values are also been determined for most common medicinal plants in the study area. Cf values of medicinal plants were estimated using the formula:(number of people interviewed citing species/the total number of people interviewed) x100 (Friedman et al. 1986).

\section{Results and Discussion}

A total of 92 ethnomedicinal plant species under 46 families have been recorded from the study area. These are used against 55 ailments through 200 formularies. The present results are the indication of rich ethnomedicinal plants with use diversity in the study area. Though Dhaka city is devoid of natural forests nowadays but urban people have still some link with natural ethnomedicinal plants for their primary healthcare. This is proved from the current research results. Apart from this explanation community living around Dhaka city is still in primitive mode of lifestyle in practices. For each species scientific name, local name, family, parts used, ailments and mode of treatment are presented (Table 1). Among the total plants, 11 species have been used for diabetes management by the community people (Table 2 ).

Table 1. Diversity of ethnomedicinal plants with local uses in the study area.

\begin{tabular}{|c|c|c|c|c|c|}
\hline Scientific name & Local name & Family & $\begin{array}{l}\text { Parts } \\
\text { used }\end{array}$ & Ailments & Treatment mode \\
\hline \multirow[t]{2}{*}{$\begin{array}{l}\text { Abroma augusta (L.) L. f., Z- } \\
68\end{array}$} & Ulotkambal & Sterculiaceae & Stem & Constipation & $\begin{array}{l}\text { Stem soaked in water for } \\
\text { whole night then the extract } \\
\text { is taken }\end{array}$ \\
\hline & & & Leaves & Body cooling & Juice is taken \\
\hline $\begin{array}{l}\text { Acacia catechu (L. f.) Willd., } \\
\text { Z-41 }\end{array}$ & Khoirkata & Mimosaceae & Root & Dysentery & Root juice is taken \\
\hline Achyranthes aspera L., Z-22 & Apang & Amaranthaceae & Root & Jaundice & Root juice is taken \\
\hline Acorus calamus L., Z-39 & Boss & Araceae & Root & $\begin{array}{l}\text { Reduce } \\
\text { cholesterol }\end{array}$ & Cooked root is eaten \\
\hline \multirow{3}{*}{$\begin{array}{l}\text { Adhatoda zeylanica Medic., } \\
\text { Z-07 }\end{array}$} & Bashok & Acanthaceae & Leaves & Cough & Leaves chewed directly \\
\hline & & & & Cough and Cold & Decoction is taken \\
\hline & & & & & $\begin{array}{l}\text { Leaf juice mixed with basil } \\
\text { is taken } 1 \text { spoon each time }\end{array}$ \\
\hline $\begin{array}{l}\text { Aegle marmelos (L.) Corr., } \\
\text { Z-52 }\end{array}$ & Bel & Rutaceae & Fruit & Dysentery & Juice is taken \\
\hline Allium sativum L., Z-58 & Roshun & Liliaceae & Bulb & Pain & $\begin{array}{l}\text { One cube is taken in the } \\
\text { evening }\end{array}$ \\
\hline
\end{tabular}


Table 1 contd.

\begin{tabular}{|c|c|c|c|c|c|}
\hline Scientific name & Local name & Family & $\begin{array}{l}\text { Parts } \\
\text { used }\end{array}$ & Ailments & Treatment mode \\
\hline & & & Bulb & Heart disease & Bulb is eaten in empty stomach \\
\hline & & & & Tonic & Juice is taken \\
\hline \multirow[t]{8}{*}{ Aloe vera (L.) Burm. f., Z-13 } & Alovera & Crassulaceae & Leaves & Stomachache & Leaf juice is taken \\
\hline & & & & Dysentery & Inner mucilage of leaves is taken \\
\hline & & & & Diabetes & Inner mucilage of leaves is taken \\
\hline & & & Latex & Skin disease & Latex juice is applied \\
\hline & & & & Jaundice & Latex juice is taken \\
\hline & & & & Hair treatment & Paste is applied \\
\hline & & & & Diabetes & Latex is taken \\
\hline & & & & Constipation & Latex is taken \\
\hline $\begin{array}{l}\text { Alternanthera sessilis (L.) R. } \\
\text { Br.ex Roem \& Schult., Z-70 }\end{array}$ & Haicha & Amaranthaceae & Leaves & Antioxidant & Cooked leaves are taken \\
\hline \multirow{2}{*}{$\begin{array}{l}\text { Amaranthus spinosus L. , Z- } \\
74\end{array}$} & Katanote & Amaranthaceae & Root & Menstruation & Root juice is taken \\
\hline & & & & Diabetes & Root is eaten for 1 month \\
\hline \multirow{2}{*}{$\begin{array}{l}\text { Anacardium occidentale L., } \\
\text { Z-62 }\end{array}$} & Kajubadam & Anacardiaceae & Fruit & Heart disease & Fruits are eaten directly \\
\hline & & & & Bone fracture & Fruit is eaten directly \\
\hline \multirow{4}{*}{$\begin{array}{l}\text { Andrographis paniculata } \\
\text { (Burm. f.) Wall. ex Nees, Z- } \\
08\end{array}$} & Kalomegh & Acanthaceae & Leaves & $\begin{array}{l}\text { Female } \\
\text { disease }\end{array}$ & Juice is taken \\
\hline & & & & Cough & Leaves are chewed \\
\hline & & & & Cold & Leaves are chewed \\
\hline & & & & Blood purifier & Paste is taken \\
\hline Areca catechu L., Z-110 & Supari & Arecaceae & Fruit & Heart disease & Raw young fruit is taken \\
\hline \multirow{2}{*}{$\begin{array}{l}\text { Artocarpus heterophyllus } \\
\text { Lamk., Z-78 }\end{array}$} & Kathal & Moraceae & Latex & Ring worm & Latex is applied directly \\
\hline & & & Fruit & Antioxidant & Fruit is eaten \\
\hline \multirow[t]{2}{*}{$\begin{array}{l}\text { Asparagus racemosus } \\
\text { Willd., Z-19 }\end{array}$} & Shatamuli & Liliaceae & Stem & $\begin{array}{l}\text { Liver } \\
\text { complain }\end{array}$ & Inner part is eaten \\
\hline & & & Root & Gastritis & Powdered root is taken \\
\hline \multirow{6}{*}{$\begin{array}{l}\text { Azadirachta indica A. Juss., } \\
\mathrm{Z}-47\end{array}$} & Neem & Meliaceae & Leaves & Stomach pain & Powder is taken \\
\hline & & & & Skin disease & Decoction is used for taking bath \\
\hline & & & & Pesticide & Decoction is sprayed \\
\hline & & & & $\begin{array}{l}\text { High blood } \\
\text { pressure }\end{array}$ & Leaves powder is taken \\
\hline & & & & Hair treatment & Paste is applied for killing lice \\
\hline & & & & Diabetes & Juice is taken \\
\hline $\begin{array}{l}\text { Baccaurea ramiflora Lour. } \\
\text { Z-102 }\end{array}$ & Lotkon & Euphorbiaceae & Fruit & $\begin{array}{l}\text { Cold } \\
\text { treatment }\end{array}$ & Juice is taken \\
\hline
\end{tabular}


Table 1 contd.

\begin{tabular}{|c|c|c|c|c|c|}
\hline Scientific name & Local name & Family & $\begin{array}{l}\text { Parts } \\
\text { used }\end{array}$ & Ailments & Treatment mode \\
\hline \multirow[t]{2}{*}{ Bombax ceiba L. , Z-48 } & Shimul & Bombacaceae & Root & Gonorrhoea & $\begin{array}{l}\text { Cold extract is taken in empty } \\
\text { stomach in the morning }\end{array}$ \\
\hline & & & & $\begin{array}{l}\text { Calcium } \\
\text { deficiency }\end{array}$ & Inner part is taken \\
\hline $\begin{array}{l}\text { Brassica campestris L., } \\
\text { Z-61 }\end{array}$ & Ryesorisa & Brassicaceae & Seed & Skin disease & Seed oil is applied \\
\hline \multirow{12}{*}{$\begin{array}{l}\text { Bryophyllum pinnatum } \\
\text { (Lam.) Oken , Z-27 }\end{array}$} & Pathorkuchi & Crassulaceae & Leaves & Stomach pain & Leaves are chewed \\
\hline & & & & Joint pain & Leaves juice is taken \\
\hline & & & & Gastritis & $\begin{array}{l}\text { Leaves are chewed three times in a } \\
\text { day }\end{array}$ \\
\hline & & & & Gallbladder & Juice is taken \\
\hline & & & & Dysentery & Leaves are chewed \\
\hline & & & & $\begin{array}{l}\text { Cuts and } \\
\text { wounds }\end{array}$ & Paste is applied \\
\hline & & & & Constipation & Leaves are chewed \\
\hline & & & & Cold & Paste is applied \\
\hline & & & & Cholera & Paste is applied \\
\hline & & & & Acne & Paste is applied directly \\
\hline & & & $\begin{array}{l}\text { Whole } \\
\text { plant }\end{array}$ & Diabetes & Juice is taken \\
\hline & & & & Jaundice & Leaf juice is taken \\
\hline \multirow{2}{*}{$\begin{array}{l}\text { Cajanus cajan }(\mathrm{L} .) \\
\text { Millsp., Z-85 }\end{array}$} & Orhor & Fabaceae & Leaves & Jaundice & Juice is taken in empty stomach \\
\hline & & & & & $\begin{array}{l}\text { Cold extract is taken } \\
\text { Juice is taken }\end{array}$ \\
\hline $\begin{array}{l}\text { Calotropis gigantea (L.) } \\
\text { R. Br., Z-55 }\end{array}$ & Akanda & Asclepiadaceae & Leaves & Pain & Juice is taken \\
\hline \multirow[t]{2}{*}{$\begin{array}{l}\text { Catharanthus roseus (L.) } \\
\text { G. Don, Z-14 }\end{array}$} & Noyontara & Apocynaceae & Flower & Diabetes & $\begin{array}{l}\text { Flowers are chewed in empty } \\
\text { stomach twice per day }\end{array}$ \\
\hline & & & Leaves & & $\begin{array}{l}\text { Half cup leaves juice is taken } \\
\text { twice per day }\end{array}$ \\
\hline Cassia alata L., Z-29 & Dadmardan & Caesalpiniaceae & Leaves & Eczema & Leaves juice is taken \\
\hline \multirow[t]{7}{*}{$\begin{array}{l}\text { Centella asiatica (L.), } \\
\text { Urban, Z-106 }\end{array}$} & Manik pata & Apiaceae & Leaves & $\begin{array}{l}\text { Stomach } \\
\text { treatment }\end{array}$ & Juice is taken \\
\hline & & & & Stomach pain & $\begin{array}{l}\text { Leaves paste is taken with ginger } \\
\text { and salt }\end{array}$ \\
\hline & & & & Dysentery & Juice is taken in empty stomach \\
\hline & & & & Diarrhoea & Juice is taken \\
\hline & & & & & Leaves juice is taken \\
\hline & & & & Constipation & Leaves paste is taken \\
\hline & & & & Brain promoting & Leaves are chewed \\
\hline $\begin{array}{l}\text { Cinnamomum camphora } \\
\text { (L.) J. Presl, Z-63 }\end{array}$ & Korpur & Lauraceae & Fruit & Antibacterial & Fruits are taken \\
\hline \multirow{2}{*}{$\begin{array}{l}\text { Clerodendrum viscosum } \\
\text { Vent., Z-15 }\end{array}$} & Vat & Verbenaceae & Leaves & Liver control & 4-5 leaves are chewed \\
\hline & & & & Worm & Leaves paste is taken until cure \\
\hline
\end{tabular}


Table 1 contd.

\begin{tabular}{|c|c|c|c|c|c|}
\hline Scientific name & Local name & Family & $\begin{array}{l}\text { Parts } \\
\text { used }\end{array}$ & Ailments & Treatment mode \\
\hline \multirow{3}{*}{$\begin{array}{l}\text { Coccinia grandis (L.) } \\
\text { Voigt, Z-01 }\end{array}$} & Telakucha & Cucurbitaceae & Leaves & Stomach pain & Cooked leaves are eaten \\
\hline & & & & Diabetes & $\begin{array}{l}\text { Leaves are chewed } \\
\text { Leaves juice is taken } \\
\text { Cooked leaves are eaten }\end{array}$ \\
\hline & & & Fruit & Diabetes & Cooked fruits are eaten \\
\hline \multirow[t]{3}{*}{ Cocos nucifera L., Z-99 } & Dab & Arecaceae & Fruit & Body cooling & Fruit juice is taken \\
\hline & & & & Jaundice & Juice is taken \\
\hline & & & & Digestion & Juice is taken \\
\hline \multirow{4}{*}{$\begin{array}{l}\text { Colocasia esculenta (L.) } \\
\text { Schott, Z-107 }\end{array}$} & Kochu & Araceae & Leaves & Snake bite & Paste is applied directly \\
\hline & & & & & Paste is applied directly \\
\hline & & & & Chest burning & Cooked leaves are taken \\
\hline & & & Flower & Body pain & Cooked flowers are eaten \\
\hline Crinum asiaticum L., Z-45 & Bonrosun & Liliaceae & Root & Asthma & Juice is taken \\
\hline \multirow[t]{3}{*}{ Cuscuta reflexa Roxb., Z-16 } & Sunnalota & Cuscutaceae & $\begin{array}{l}\text { Whole } \\
\text { plant }\end{array}$ & Jaundice & $\begin{array}{l}\text { Juice is taken one spoonful } \\
\text { twice a day }\end{array}$ \\
\hline & & & & Worm & $\begin{array}{l}\text { Juice is taken one spoonful } \\
\text { twice a day }\end{array}$ \\
\hline & & & Leaves & Jaundice & Juice is taken \\
\hline \multirow{2}{*}{$\begin{array}{l}\text { Cynodon dactylon (L.) } \\
\text { Pers., Z-53 }\end{array}$} & Durba grass & Poaceae & Leaves & Diabetes & Juice is taken \\
\hline & & & & $\begin{array}{l}\text { Cuts and } \\
\text { wounds }\end{array}$ & Paste is applied directly \\
\hline \multirow[t]{2}{*}{ Datura metel L., Z-10 } & Datura & Solanaceae & Leaves & Skin disease & $\begin{array}{l}\text { Cooked leaves are taken } \\
\text { Leaves paste is applied }\end{array}$ \\
\hline & & & & Madness & Leaves juice is taken \\
\hline \multirow[t]{2}{*}{$\begin{array}{l}\text { Eclipta prostrata }(\mathrm{L} .) \\
\text { Mant., Z-31 }\end{array}$} & Keshoraj & Asteraceae & Leaves & Hair treatment & Paste leaves is applied \\
\hline & & & & & Leaves juice is applied to hair \\
\hline $\begin{array}{l}\text { Eupatorium odoratum L., } \\
\text { Z-43 }\end{array}$ & Fulkuri & Asteraceae & Leaves & $\begin{array}{l}\text { Cuts and } \\
\text { wounds }\end{array}$ & Leaves paste is applied \\
\hline Ficus racemosa L., Z-72 & Jogdumur & Moraceae & Fruit & Diabetes & Fruits are eaten \\
\hline $\begin{array}{l}\text { Glinus oppositifolius (L.) A. } \\
\text { DC., Z-71 }\end{array}$ & Geemashak & Molluginaceae & Leaves & Antioxidant & Cooked leaves are taken \\
\hline $\begin{array}{l}\text { Glycosmis arborea (Roxb.) } \\
\text { A. DC. , Z-108 }\end{array}$ & Motkila & Rutaceae & Leaves & Worm & Leaves are juice applied \\
\hline \multirow{4}{*}{$\begin{array}{l}\text { Gynura nepalensis DC., Z- } \\
25\end{array}$} & Gynura & Asteraceae & Leaves & Diabetes & Leaves juice is taken \\
\hline & & & & & $\begin{array}{l}\text { Leaves are chewed morning } \\
\text { and afternoon }\end{array}$ \\
\hline & & & & & $\begin{array}{l}\text { Leaves are chewed in the } \\
\text { morning and evening }\end{array}$ \\
\hline & & & & & 2-3 leaves are chewed \\
\hline \multirow{2}{*}{$\begin{array}{l}\text { Heliotropium indicum L., Z- } \\
18\end{array}$} & Hathishur & Boraginaceae & Leaves & Insect bite & Paste is applied directly \\
\hline & & & & Eye treatment & Leaves juice is taken \\
\hline
\end{tabular}


Table 1 contd.

\begin{tabular}{|c|c|c|c|c|c|}
\hline Scientific name & Local name & Family & Parts used & Ailments & Treatment mode \\
\hline \multirow{6}{*}{$\begin{array}{l}\text { Hibiscus rosa sinensis L., } \\
\text { Z-51 }\end{array}$} & Roktojoba & Malvaceae & Flower & Women disease & Cold extract is taken \\
\hline & & & & Menstruation & $\begin{array}{l}\text { Cold extract is taken for } 1 \\
\text { week }\end{array}$ \\
\hline & & & & Dysentery & Flowers are taken \\
\hline & & & Leaves & Fever & Leaves are chewed \\
\hline & & & & Diabetes & $\begin{array}{l}\text { Juice of } 5 \text { leaves is taken in } \\
\text { the morning and night }\end{array}$ \\
\hline & & & & & $\begin{array}{l}\text { Powder of seed, bark of } \\
\text { arjun, fenugreek, tokma, and } \\
\text { usufgul are taken }\end{array}$ \\
\hline $\begin{array}{l}\text { Hygrophila auriculata } \\
\text { (Schum.) Heine, Z-93 }\end{array}$ & Talmakhna & Acanthaceae & Fruit & $\begin{array}{l}\text { Kidney } \\
\text { treatment }\end{array}$ & $\begin{array}{l}\text { Juice is taken in empty } \\
\text { stomach in the morning and } \\
\text { afternoon }\end{array}$ \\
\hline $\begin{array}{l}\text { Hylocereus undatus } \\
\text { (Haworth) Britton \& } \\
\text { Rose, Z-111 }\end{array}$ & Dragon fruit & Cactaceae & Fruit & Heart disease & Fruit is taken \\
\hline \multirow{2}{*}{$\begin{array}{l}\text { Hyptis suaveolens (L.) } \\
\text { Poit., Z-100 }\end{array}$} & Tokma & Lamiaceae & Leaves & Stomachache & Leaves juice is taken \\
\hline & & & Seed & Body cooling & Cold extract is taken \\
\hline $\begin{array}{l}\text { Ipomoea aquatica } \\
\text { Forssk., Z-105 }\end{array}$ & Kalmisak & Convolvulaceae & Leaves & Antioxidant & Cooked leaves are eaten \\
\hline \multirow[t]{2}{*}{$\begin{array}{l}\text { Lannea coromandelica } \\
\text { (Houtt.) Merr., Z-103 }\end{array}$} & Jiga & Anacardiaceae & Bark & Dysentery & $\begin{array}{l}\text { Juice is taken with green } \\
\text { banana, guava and barks of } \\
\text { mango and stone apple }\end{array}$ \\
\hline & & & & Constipation & Cold extract is taken \\
\hline $\begin{array}{l}\text { Leucas aspera (Willd.) } \\
\text { Link, Z-80 }\end{array}$ & Dondokolosh & Lamiaceae & Leaves & Cold treatment & Cooked leaves are taken \\
\hline $\begin{array}{l}\text { Litsea glutinosa (Lour.) } \\
\text { Robinson, Z-28 }\end{array}$ & Peepul tree & Lauraceae & Leaves & Diabetes & Decoction is taken \\
\hline $\begin{array}{l}\text { Mangifera indica (L.), Z- } \\
73\end{array}$ & Aam & Anacardiaceae & $\begin{array}{l}\text { Young } \\
\text { leaf }\end{array}$ & Diabetes & Juice is taken \\
\hline Mentha arvensis L. , Z-17 & Pudina & Lamiaceae & Leaves & Cold treatment & Leaves juice is taken \\
\hline \multirow{2}{*}{$\begin{array}{l}\text { Mikania cordata (Burm. } \\
\text { f.)Robinson, Z-83 }\end{array}$} & Japanilata & Asteraceae & Leaves & Cuts and wounds & Paste is applied \\
\hline & & & Stem & Cuts and wounds & Paste is applied \\
\hline \multirow[t]{2}{*}{ Mimosa pudica L., Z-02 } & Lajjabati & Mimosaceae & Stem & Women disease & Root is applied \\
\hline & & & Leaves & Heart disease & Cold extract is taken \\
\hline Mirabilis jalapa L., Z-59 & Sondhamalati & Nyctaginaceae & Flower & Cold treatment & Flowers are eaten raw \\
\hline \multirow[t]{2}{*}{$\begin{array}{l}\text { Momordica dioica Roxb. } \\
\text { ex. Willd., Z-46 }\end{array}$} & Titakorolla & Cucurbitaceae & Fruit & Diabetes & $\begin{array}{l}\text { Juice is taken in empty } \\
\text { stomach }\end{array}$ \\
\hline & & & & & Cold extract is taken \\
\hline \multirow[t]{4}{*}{$\begin{array}{l}\text { Moringa oleifera Lam., Z- } \\
65\end{array}$} & Shajna & Moringaceae & Leaves & Pain & $\begin{array}{l}\text { Paste is taken with black } \\
\text { cumin and garlic }\end{array}$ \\
\hline & & & & Deworming & Paste is taken \\
\hline & & & & Constipation & $\begin{array}{l}\text { Cooked drumstick is taken } \\
\text { with black cumin and } \\
\text { garlic }\end{array}$ \\
\hline & & & Fruit & Constipation & Cooked fruit is eaten \\
\hline
\end{tabular}


Table 1 contd.

\begin{tabular}{|c|c|c|c|c|c|}
\hline Scientific name & Local name & Family & $\begin{array}{l}\text { Parts } \\
\text { used }\end{array}$ & Ailments & Treatment mode \\
\hline $\begin{array}{l}\text { Murraya koenigii (L.) } \\
\text { Spreng., Z-75 }\end{array}$ & Kamini & Rutaceae & Leaves & Toothache & Decoction is taken \\
\hline \multirow[t]{6}{*}{ Musa sapientum L., Z-50 } & Kacha kola & Musaceae & Latex & Skin disease & Latex is applied \\
\hline & & & $\begin{array}{l}\text { Inner } \\
\text { part }\end{array}$ & Heart disease & Cooked inner part is taken \\
\hline & & & & Gastric & $\begin{array}{l}1 \text { fruit with } 4 / 5 \text { bed bugs is } \\
\text { taken }\end{array}$ \\
\hline & & & & Diabetes & Juice is taken \\
\hline & & & Fruit & Digestion & Juice is taken \\
\hline & & & & Cold treatment & Fruits are taken directly \\
\hline \multirow[t]{2}{*}{ Nigella sativa L., Z-49 } & Kalojira & Apiaceae & Fruit & Heart disease & $\begin{array}{l}\text { Black cumin with honey is } \\
\text { taken for } 1 \text { month }\end{array}$ \\
\hline & & & & Hair treatment & Paste is applied \\
\hline $\begin{array}{l}\text { Ocimum gratissimum L., } \\
\text { Z-06 }\end{array}$ & Ramtulsi & Lamiaceae & Leaves & Asthma & Leaves juice is taken \\
\hline \multirow[t]{2}{*}{ Ocimum sanctum $\mathrm{L} ., \mathrm{Z}-05$} & Tulsi & Lamiaceae & Leaves & Cough & Leaves are chewed \\
\hline & & & & Cold treatment & Young leaves juice is taken \\
\hline Paederia foetida L., Z-32 & Gondhovadali & Rubiaceae & Leaves & Dysentery & Leaves juice is taken \\
\hline $\begin{array}{l}\text { Phyllanthus emblica L., } \\
\text { Z-95 }\end{array}$ & Aamloki & Euphorbiaceae & Fruit & Heart disease & $\begin{array}{l}\text { Mixture of amla, myrobalan } \\
\text { and beleric cold extract are } \\
\text { taken }\end{array}$ \\
\hline $\begin{array}{l}\text { Phyllanthus reticulatus } \\
\text { Poir. , Z-104 }\end{array}$ & Chitki & Euphorbiaceae & Leaves & Diabetes & Cold extract is taken \\
\hline \multirow[t]{3}{*}{$\begin{array}{l}\text { Plantago ovata Forssk., } \\
\text { Z-88 }\end{array}$} & Usufgul & Plantaginaceae & $\begin{array}{l}\text { Seed } \\
\text { coat }\end{array}$ & Pressure & Cold extract is taken \\
\hline & & & & Diabetes & Cold extract is taken \\
\hline & & & & Constipation & Cold extract of seeds is taken \\
\hline \multirow{2}{*}{$\begin{array}{l}\text { Psidium guajava } \text { L. Bat., } \\
\text { Z-67 }\end{array}$} & Peara & Myrtaceae & Leaves & Toothache & Decoction is applied \\
\hline & & & Fruit & Toothache & Fruits are eaten \\
\hline \multirow[t]{4}{*}{$\begin{array}{l}\text { Rauvolfia serpentina (L.) } \\
\text { Benth. ex Kurz, Z-04 }\end{array}$} & Sharpagandha & Apocynaceae & Root & $\begin{array}{l}\text { Madness } \\
\text { treatment }\end{array}$ & Root juice is taken \\
\hline & & & & Heart disease & $\begin{array}{l}\text { Powder is taken after meal } \\
\text { twice per day }\end{array}$ \\
\hline & & & & Deworming & $\begin{array}{l}\text { Powder is taken after meal } \\
\text { twice per day }\end{array}$ \\
\hline & & & & Blood pressure & $\begin{array}{l}\text { Powder is taken after meal } \\
\text { twice per day }\end{array}$ \\
\hline $\begin{array}{l}\text { Saccharum spontaneum } \\
\text { L., Z-40 }\end{array}$ & Gandari & Poaceae & $\begin{array}{l}\text { Whole } \\
\text { plant }\end{array}$ & Jaundice & $\begin{array}{l}\text { One glass juice is taken } \\
\text { twice per day }\end{array}$ \\
\hline $\begin{array}{l}\text { Saraca asoca (Roxb.) de } \\
\text { Wild., Z-26 }\end{array}$ & Ashok & Fabaceae & Bark & Anti leukemia & $\begin{array}{l}\text { Cold extract is taken in empty } \\
\text { stomach }\end{array}$ \\
\hline \multirow[t]{3}{*}{ Scoparia dulcis L., Z-34 } & Chinigura & Scrophulariaceae & $\begin{array}{l}\text { Whole } \\
\text { plant }\end{array}$ & Dysentery & Juice is taken \\
\hline & & & & Diabetes & $\begin{array}{l}\text { Powder is taken in empty } \\
\text { stomach in the morning }\end{array}$ \\
\hline & & & Leaves & Diarrhoea & Juice is taken \\
\hline
\end{tabular}


Table 1 contd.

\begin{tabular}{|c|c|c|c|c|c|}
\hline Scientific name & Local name & Family & $\begin{array}{l}\text { Parts } \\
\text { used }\end{array}$ & Ailments & Treatment mode \\
\hline & & & & All disease & Cooked leaves are taken \\
\hline \multirow{2}{*}{$\begin{array}{l}\text { Senna alexandrina Mill., } \\
\text { Z-97 }\end{array}$} & Sonapata & Caesalpiniaceae & Leaves & Gastric & Cold extract is taken \\
\hline & & & & Weight loss & Cold extract is taken \\
\hline $\begin{array}{l}\text { Spondias pinnata (L. f.) } \\
\text { Kurz, Z-87 }\end{array}$ & Amra & Anacardiaceae & Fruit & Heart disease & Fruits are eaten \\
\hline \multirow[t]{4}{*}{ Streblus asper Lour., Z-42 } & Sheora & Moraceae & Leaves & Diabetes & Leaves juice is taken \\
\hline & & & & Bone fracture & Cooked leaves are taken \\
\hline & & & Root & Bone fracture & Root paste is applied \\
\hline & & & Latex & Acne & Latex is applied \\
\hline $\begin{array}{l}\text { Strychnos nux-vomica L. } \\
\text {, Z-84 }\end{array}$ & Kuchila & Loganiaceae & Leaves & Jaundice & Cold extract is taken \\
\hline \multirow[t]{2}{*}{ Swertia perennis $\mathrm{L}$. } & Chirata & Gentianaceae & Leaves & Fever & Cold extract is taken \\
\hline & & & & Blood purifier & Paste is taken \\
\hline \multirow{4}{*}{$\begin{array}{l}\text { Swietenia mahagoni (L.) } \\
\text { Jacq, Z-03 }\end{array}$} & Mahogany & Meliaceae & Seed & Pesticide & Cold extract is sprayed \\
\hline & & & & Joint pain & $\begin{array}{l}\text { Crushed seed is taken after } \\
\text { meal twice per day }\end{array}$ \\
\hline & & & & Diabetes & $\begin{array}{l}\text { Juice is taken in empty } \\
\text { stomach }\end{array}$ \\
\hline & & & & & $\begin{array}{l}\text { Crushed seed mixed with } \\
\text { fenugreek is taken twice per } \\
\text { day }\end{array}$ \\
\hline \multirow{3}{*}{$\begin{array}{l}\text { Syzygium cumini (L.) } \\
\text { Skeels, Z-82 }\end{array}$} & Kalojam & Myrtaceae & Seed & Jaundice & Juice is taken \\
\hline & & & & Diabetes & $\begin{array}{l}\text { Powder of black berry with } \\
\text { fenugreek fruits are mixed } \\
\text { with water and then is taken } \\
1 \text { teaspoon once a day, fruit } \\
\text { taken directly }\end{array}$ \\
\hline & & & Leaves & & Leaves are chewed \\
\hline Tagetes patula L., Z-101 & Gada & Asteraceae & Leaves & Antiseptic & Leaf paste is applied \\
\hline \multirow{3}{*}{$\begin{array}{l}\text { Tamarindus indica L., Z- } \\
66\end{array}$} & Tetul & Caesalpiniaceae & Fruit & Urine infection & Cold extract is taken \\
\hline & & & & High pressure & Fruit juice is taken \\
\hline & & & & Diabetes & $\begin{array}{l}\text { Juice is taken in empty } \\
\text { stomach }\end{array}$ \\
\hline \multirow{2}{*}{$\begin{array}{l}\text { Terminalia arjuna (Roxb. } \\
\text { ex DC.) Wight \& Arn., Z- } \\
09\end{array}$} & Arjun & Combretaceae & Bark & High pressure & $\begin{array}{l}\text { Juice is taken in empty } \\
\text { stomach }\end{array}$ \\
\hline & & & & Heart disease & $\begin{array}{l}\text { Powdered bark is taken in } \\
\text { empty stomach early in the } \\
\text { morning }\end{array}$ \\
\hline
\end{tabular}


Table 1 contd.

\begin{tabular}{|c|c|c|c|c|c|}
\hline Scientific name & Local name & Family & $\begin{array}{l}\text { Parts } \\
\text { used }\end{array}$ & Ailments & Treatment mode \\
\hline & & & & Heart disease & $\begin{array}{l}\text { Powdered bark mixed with } \\
\text { amla and beleric then is } \\
\text { taken } 1 \text { spoon twice per day }\end{array}$ \\
\hline $\begin{array}{l}\text { Terminalia bellirica } \\
\text { (Gaertn.) Roxb., Z-109 }\end{array}$ & Bohera & Combretaceae & Fruit & Gastric & $\begin{array}{l}\text { Cold extract is taken in the } \\
\text { morning }\end{array}$ \\
\hline $\begin{array}{l}\text { Terminalia citrina } \\
\text { (Gaertn.) Roxb. ex } \\
\text { Fleming, Z-94 }\end{array}$ & Horitaki & Combretaceae & Fruit & Heart disease & $\begin{array}{l}\text { Cold extract is taken in the } \\
\text { morning }\end{array}$ \\
\hline $\begin{array}{l}\text { Tinospora cordifolia } \\
\text { (Wild.) Hook. f. \& } \\
\text { Thoms., Z-91 }\end{array}$ & Aamkuruj & Menispermaceae & Stem & Diabetes & Cold extract is taken \\
\hline $\begin{array}{l}\text { Trigonella foenum- } \\
\text { graeceum L., Z-81 }\end{array}$ & Methi & Fabaceae & Leaves & Diabetes & Cold extract is taken \\
\hline Vitex negundo L., Z-24 & Nishinda & Verbenaceae & Leaves & Insomnia & Leaves are kept under pillow \\
\hline $\begin{array}{l}\text { Vitis quadrangularis } \\
\text { Wall. ex Wight \& Arn., Z- } \\
60\end{array}$ & Harvanga & Vitaceae & Stem & Bone fracture & Paste is applied \\
\hline Vitis vinifera $\mathrm{L} ., \mathrm{Z}-98$ & Angur & Vitaceae & Fruit & Diabetes & $\begin{array}{l}100 \mathrm{gm} \text { is taken in the } \\
\text { morning and night }\end{array}$ \\
\hline $\begin{array}{l}\text { Wedelia chinensis } \\
\text { (Osbeck) Merr., Z-37 }\end{array}$ & Vingoraj & Asteraceae & Leaves & Hair treatment & Leaves paste is applied \\
\hline $\begin{array}{l}\text { Withania somnifera (L.) } \\
\text { Dunal, Z-44 }\end{array}$ & Orshogondha & Solanaceae & Root & Energy tonic & Root powder is taken \\
\hline $\begin{array}{l}\text { Xanthosoma violaceum } \\
\text { Schott, Z-64 }\end{array}$ & Dudkachu & Araceae & Leaves & Diabetes & Cooked leaves are taken \\
\hline $\begin{array}{l}\text { Zanthoxylum rhetsa } \\
\text { (Roxb.) DC., Z-96 }\end{array}$ & Bajna & Rutaceae & Fruit & $\begin{array}{l}\text { Gastric, } \\
\text { Weakness }\end{array}$ & $\begin{array}{l}\text { One glass of juice is taken, } \\
\text { Oil is taken }\end{array}$ \\
\hline $\begin{array}{l}\text { Zingiber officinale Rosc., } \\
\text { Z-54 }\end{array}$ & Ada & Zingiberaceae & Rhizome & Gastric & Juice is taken \\
\hline
\end{tabular}

The medicinal plants used for the diabetes management are not equally important. Some species are more important than others. Widely cited species for particular purpose by the community people are considered popular species. According to community people most cited medicinal plant species for the diabetes management are Gynura nepalensis DC., Coccinia grandis L. Voigt, Aloe vera (L.) Burm. f., Syzygium cumini (L.) Skeels, Swietenia mahagoni (L.) Jacq Momordica dioica Roxb. ex. Willd., Catharanthus roseus (L.) G. Don, Streblus asper Lour., Bryophyllum pinnatum (Lamk.) Oken, Tamarindus indica L. and Scoparia dulcis L. Such citations are the indication of importance of ethnomedicinal plants in the study area. Most cited species are also good candidate for further ethnopharmacology studies to find new compounds.

Life form of medicinal plant species showed variations. In our study herbs are the common ethnomedicinal plants followed by trees, shrubs and climbers (Fig. 1). This pattern of life forms was found in the previous research (Uddin et al. 2017). In case of parts used, leaf is always dominant parts used for the preparation of herbal medicine by community people found in the present study (Fig. 2). Such use trend is the indication of sustainable resource exploitation from the nature. 
Table 2. Most cited medicinal plant species for diabetes management.

\begin{tabular}{|c|c|c|c|c|c|}
\hline Species & Local name & $\begin{array}{l}\text { Parts } \\
\text { used }\end{array}$ & Ailments & Treatment mode & Citation \\
\hline $\begin{array}{l}\text { Gynura nepalensis } \\
\text { DC., Z-25 }\end{array}$ & Gynura & Leaves & Diabetes & $\begin{array}{l}\text { Leaf juice is taken, Leaves are } \\
\text { chewed morning and afternoon }\end{array}$ & 103 \\
\hline $\begin{array}{l}\text { Coccinia grandis } \mathrm{L} \\
\text {.Voigt., Z- } 01\end{array}$ & Telakucha & $\begin{array}{l}\text { Leaves, } \\
\text { Fruit }\end{array}$ & Diabetes & $\begin{array}{l}\text { Leaves are chewed directly, } \\
\text { Leaf juice is taken, cooked } \\
\text { leaves area eaten, cooked fruits } \\
\text { are eaten }\end{array}$ & 100 \\
\hline $\begin{array}{l}\text { Aloe vera (L.) Burm. } \\
\text { f., Z-13 }\end{array}$ & Alovera & Leaves & Diabetes & $\begin{array}{l}\text { Inner mucilage of leaves is } \\
\text { taken }\end{array}$ & 60 \\
\hline $\begin{array}{l}\text { Syzygium cumini (L.) } \\
\text { Skeels, Z-82 }\end{array}$ & Kalojam & $\begin{array}{l}\text { Leaves, } \\
\text { fruit, } \\
\text { Seed }\end{array}$ & Diabetes & $\begin{array}{l}\text { Leaves are chewed, pulp of } \\
\text { fruit is taken, powder of seed } \\
\text { mixed with water is taken }\end{array}$ & 45 \\
\hline $\begin{array}{l}\text { Swietenia mahagoni } \\
\text { (L.) Jacq, Z-03 }\end{array}$ & Mahogany & Seed & Diabetes & $\begin{array}{l}\text { Seed powder is taken in empty } \\
\text { stomach, Crushed seed mixed } \\
\text { with fenugreek and is taken } \\
\text { twice per day }\end{array}$ & 44 \\
\hline $\begin{array}{l}\text { Momordica dioica } \\
\text { Roxb. ex. Willd., Z-46 }\end{array}$ & Korla & Fruit & Diabetes & Juice is taken in empty stomach & 42 \\
\hline $\begin{array}{l}\text { Catharanthus roseus } \\
\text { (L.) G. Don, Z-14 }\end{array}$ & Noyontara & Flower & Diabetes & $\begin{array}{l}\text { Flower is chewed in empty } \\
\text { stomach twice per day, Half } \\
\text { cup leaves juice is taken twice } \\
\text { per day }\end{array}$ & 40 \\
\hline $\begin{array}{l}\text { Streblus asper Lour., } \\
\mathrm{Z}-42\end{array}$ & sheora & Leaves & Diabetes & Leaf juice is taken & 33 \\
\hline $\begin{array}{l}\text { Bryophyllum pinnatum } \\
\text { (Lamk.) Oken , Z-27 }\end{array}$ & Pathorkuchi & Leaves & Diabetes & Juice is taken & 32 \\
\hline $\begin{array}{l}\text { Tamarindus indica L., } \\
\text { Z-66 }\end{array}$ & Tetul & $\begin{array}{l}\text { Fruit, } \\
\text { Leaves }\end{array}$ & Diabetes & $\begin{array}{l}\text { Juice is taken in empty } \\
\text { stomach, leaves are chewed }\end{array}$ & 32 \\
\hline $\begin{array}{l}\text { Scoparia dulcis L., Z- } \\
34\end{array}$ & Chinigura & Leaves & Diabetes & $\begin{array}{l}\text { Powder is taken in empty } \\
\text { stomach in the morning }\end{array}$ & 27 \\
\hline
\end{tabular}

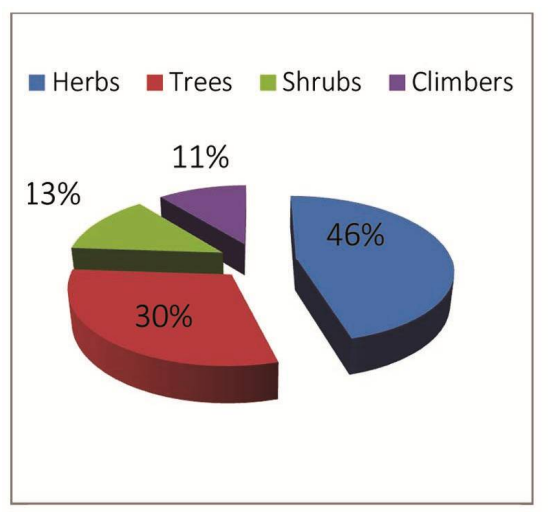

Fig. 1. Life forms ethnomedicinal plants.

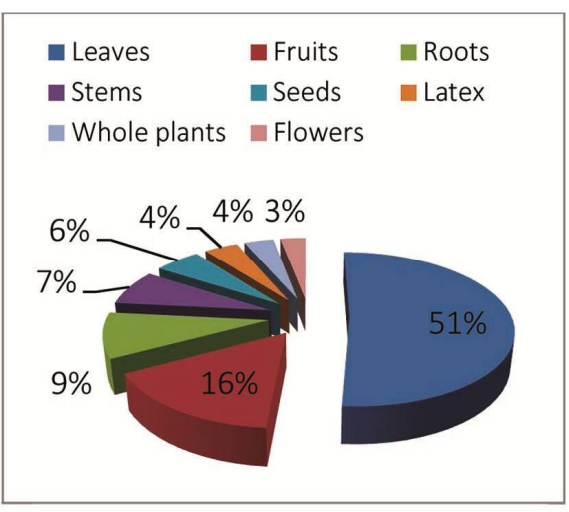

Fig. 2. Diversity of parts used. 
Fic values have been calculated to know the consensus of local people in use of ethnomedicinal plants. The disease category diabetes attained highest Fic value followed by respiratory tract, gastrointestinal tract, heart disease, dermatological, teethache, diarrhea, dysentery, hair tonic, jaundice, cuts and wound, bone, menstruation, fever and bodyache, urinary tract, impotence and others (Table 3). Diabetes is managed by 11 ethnomedicinal plant species. Most cited medicinal plants for the management of diabetes are Gynura nepalensis (Gynura), Coccinia grandis (Telakucha), Aloe vera (Alovera), Syzygium cumini (Kalojam), Swietenia mahagoni (Mahogany) and Momordica dioica (Titakorolla). These six species are very popular and in some cases are sold in the market for diabetes management.

Table 3. Values of Factor of informant consensus (Fic)

\begin{tabular}{lccc}
\hline Disease categories & Number of taxa & Used report & Fic values \\
\hline Diabetes & 11 & 589 & 0.983 \\
Respiratory tract & 12 & 232 & 0.952 \\
Gastrointestinal tract & 17 & 312 & 0.949 \\
Heart disease & 25 & 361 & 0.933 \\
Skin disease & 9 & 112 & 0.928 \\
Toothache & 2 & 13 & 0.917 \\
Diarrhoea and dysentery & 11 & 95 & 0.894 \\
Hair tonic & 6 & 48 & 0.894 \\
Jaundice & 9 & 60 & 0.864 \\
Cuts and wound & 7 & 41 & 0.85 \\
Menstruation & 5 & 21 & 0.8 \\
Fever and bodyache & 8 & 29 & 0.75 \\
Urinary tract & 2 & 3 & 0.5 \\
Impotence & 8 & 12 & 0.363 \\
Others & 15 & 86 & 0.835 \\
\hline
\end{tabular}

Gynura (Gynura nepalensis) also called diabetic plant was reported as new record from Bangladesh (Uddin et al. 2015). As Gynura proved to be an ethnomedicinal plant species for diabetes management, plantlets of this species is sold in the local market. Leaves of this plant are used by the people without further process. According to community people, the plants with bitter and sour taste are suitable for diabetes. In the present research Gynura was cited by maximum people for the management of diabetes in Dhaka city. Telakucha (Coccinia grandis) is a native wild climber growing everywhere in the roadsides and fallow lands. Due to unsustainable collection from the nature, the species has become vulnerable. As the species is important for diabetes management, distribution status in the country needs to be assessed. A further pharmacological study is necessary to find active chemical from this wonderful ethnomedicinal plant species. In our study Telakucha showed second highest citation in the use for diabetes treatment. A good number of ethnobotanical studies conducted in Bangladesh was supported Telakucha used to reduce blood sugar (Roy et al. 2008, Uddin et al. 2015, 2017). The species 
Telakucha also used for gonorrhea and skin disease (Islam et al. 2010). It is also used for burning (Roy et al., 2008).

Alovera (Aloe vera) is a wonderful herbaceous medicinal plant used in healthcare purpose from ancient time. The species is now sold in the commercial super market. A group of farmers cultivated this species in Natore Upazila. Alovera jel mixed with other medicinal plant parts is used to make energy drink in the urban footpath. Poor section of people in Dhaka city particularly rickshaw pullers, day laborers and enthusiastic people used to take this drink with faith to get relief from diabetes and other stomach related diseases and even to improve body energy. During our study a good number of people cited this species to be used in diabetes management. Alovera is also used for impotence in Bangladesh (Uddin et al. 2015, 2017). Kalojam (Syzygium cumini) is a native plant species of Bangladesh. It grows well in the natural forests and as well as homestead areas. Community people believed that fruits of Kalojam may purify human blood and seed powder also reduces blood sugar. It has been sold in the urban markets. In the present study many people cited that both fruits and seeds of Kalojam are used in diabetes management in Dhaka city. This type of result is also supported by previous research done in Bangladesh (Uddin et al. 2004). Kalojam is also used for jaundice in many parts of Bangladesh (Uddin et al. 2001, Khan et al. 2002). It is also used for toothache, and blood dysentery (Uddin et al. 2004). Mahogoni (Swietenia mahagoni) is an exotic timber yielding species naturalized in Bangladesh from long before. The plants produced oval shaped large fruits on the branch top. It contains huge number of seeds in each fruit. The pulp of the seeds is bitter in test. Community people claimed that cold extraction of seeds can reduce blood sugar but over intake may cause poison to human body. In the present study we found Mahogany was used in diabetes management by the people in and around Dhaka city. This finding is supported by previous research work done in Bangladesh (Haque et al. 2017). Titakorolla (Momordica dioica) is a very popular vegetable always sold in the all markets of Bangladesh. In our survey many people cited that Titakorolla used to manage diabetes by both urban and rural people. Same use of Titakorolla for diabetes management was reported from Feni district (Uddin et al. 2015). The present finding of Titakorolla to manage diabetes is accordance with previous findings.

During data collection we saw a good number of vendors sitting along the footpath of the Dhaka city roads and were selling crude medicinal plant parts and juice of mixture of those parts. Poor people are confident enough on such mixture and they regularly take it for caring of diabetes. In some cases, a good number of people were watching the vendor but they were refrained from take it because of hygienic question. Long term research is necessary to evaluate and validate the traditional practices displayed in the city footpath.

Observations in the field and discussions with local people, a good number of threats to ethnomedicinal plants have been identified. The most serious threats are exotic timber species plantation in and around homestead, fallow lands, roadsides and even in cultivated lands. Acacia auriculiformis, Acacia mangium, Eucalyptus camadulensis, Samanea saman, Dalbergia sissoo, Laeucaena leucocephala, Swietenia mahagoni and Cassia siamea are most preferred plant species for plantation. According to community people perception such species are very selfish plants and they do not support native species under their canopy. Medicinal plants and their traditional knowledge are in very rare in practice because of less availability in the area. As there is no written documentation on the medicinal plants, with the extinction of senior people traditional medicinal knowledge also eliminated from the urban society. They also informed us urbanization in and around Dhaka city is another challenge to ethnomedicinal plants habitats. There is ample opportunity to work on this issue but the project is very small budgeted. The current work is very preliminary. Lack of awareness among the community people is another threat to ethnomedicinal plants in the study area. People are careless to plant resources in some extend. They only care 
timber, ornamental and fruit plants. Currently they also started to care plants in roof garden. Availability of the modern medicines which promotes the negligence of use of herbal medicines among the community people in the study area is also threats to medicinal plants. Senior people with herbal knowledge do not like to share their knowledge with juniors. Due to sudden death of such people, herbal knowledge of the area lost forever.

A list of suggestion has been made based on present survey results and observations. Distribution map can be made for all culturally important medicinal plant species in the study area. Population status of such species across the habitats can be measured. Current rate of exploitation of ethnomedicinal plants by the community people could be calculated. If it seems vulnerable in the natural habitats, necessary measures for ex situ conservation could be taken. Awareness programs among the local influential persons who can make change can be created. Culturally important and most cited ethnomedicinal plants should be brought under plantation programs.

\section{Conclusion}

Diabetes is a life killing disease to the mankind. It has no permanent and final treatment in our hand. Diabetes can be controlled through management and maintenance of lifestyle activities including changing in food habit, regular exercise and proper sleeping time. In the present study documentation of total 92 ethnomedicinal plant species under 46 families in and around Dhaka city is the indication of rich ethnomedicinal plants with use diversity. Among them 11 species are used for the management of diabetes. However, all of these 11 species are not equally important in the management of diabetes. Most cited medicinal plant species for diabetes management are Gynura nepalensis DC., Coccinia grandis L. Voigt, Aloe vera (L.) Burm. f., Syzygium cumini (L.) Skeels, Swietenia mahagoni (L.) Jacq, Momordica dioica Roxb. ex. Willd., Catharanthus roseus (L.) G. Don, Streblus asper Lour., Bryophyllum pinnatum (Lamk.) Oken, Tamarindus indica L.and Scoparia dulcis L. The present result is very preliminary and based on which sound conclusion is not possible. Further ethnopharmacological study is very essential on such species to prove their efficacy in the management of diabetes. Our findings also provide baseline data to establish a connection between the traditional health practitioners and scientific communities, which could be substantial in novel drug discovery. Furthermore, ethnobotanical data is of significant value for conservation managers and policy makers for sustainable management of medicinal plant species, which are under threat due to over exploitation.

\section{Acknowledgement}

The authors acknowledge University of Dhaka and University Grants Commission Bangladesh for the financial support and also acknowledge the community people living in and around Dhaka who helped us a lot during data collection.

\section{References}

Ahmed, Z.U., Begum, Z.N.T., Hassan, M.A., Khondker, M., Kabir, S.M.H., Ahmad, M., Ahmed, A.T.A.,Rahman, A.K.A. and Haque, E.U. (Eds) 2008a. Encyclopedia of Flora and Fauna of Bangladesh, Vol. 6.Angiosperms: Dicotyledons (Acanthaceae - Asteraceae). Asiatic Society of Bangladesh, Dhaka pp. 1-408.

Ahmed, Z.U., Hassan, M.A., Begum, Z.N.T., Khondker, M., Kabir, S.M.H., Ahmad, M., Ahmed, A.T.A.,Rahman, A.K.A. and Haque, E.U. (Eds) 2008b. Encyclopedia of Flora and Fauna of Bangladesh, Vol.12. Angiosperms: Monocotyledons (Orchidaceae - Zingiberaceae). Asiatic Society of Bangladesh, Dhaka, pp. 1-552. 
Ahmed, Z.U., Hassan, M.A., Begum, Z.N.T., Khondker, M., Kabir, S.M.H., Ahmad, M., Ahmed, A.T.A.,Rahman, A.K.A. and Haque, E.U. (Eds) 2009a. Encyclopedia of Flora and Fauna of Bangladesh, Vol. 7. Angiosperms: Dicotyledons (Balsaminaceae - Euphorbiaceae). Asiatic Society of Bangladesh, Dhaka, pp. 1-546.

Ahmed, Z.U., Hassan, M.A., Begum, Z.N.T., Khondker, M., Kabir, S.M.H., Ahmad, M., Ahmed, A.T.A.,Rahman, A.K.A. and Haque, E.U. (Eds) 2009b. Encyclopedia of Flora and Fauna of Bangladesh, Vol. 8. Angiosperms: Dicotyledons (Fabaceae - Lythraceae). Asiatic Society of Bangladesh, Dhaka, pp. $1-478$.

Ahmed, Z.U., Hassan, M.A., Begum, Z.N.T., Khondker, M., Kabir, S.M.H., Ahmad, M. and Ahmed, A.T.A.(Eds) 2009c. Encyclopedia of Flora and Fauna of Bangladesh, Vol. 9. Angiosperms: Dicotyledons (Magnoliaceae - Punicaceae). Asiatic Society of Bangladesh, Dhaka, pp. 1-488.

Ahmed, Z.U., Hassan, M.A., Begum, Z.N.T., Khondker, M., Kabir, S.M.H., Ahmad, M., and Ahmed, A.T.A. (Eds) 2009d. Encyclopedia of Flora and Fauna of Bangladesh, Vol. 10. Angiosperms: Dicotyledons (Ranunculaceae - Zygophyllaceae). Asiatic Society of Bangladesh, Dhaka, pp. 1-580

Akter S., Rahman, MM, Abe SK and Sultana, P. 2014. Prevalence of diabetes and prediabetes and their risk factors among Bangladeshi adults: a nationwide survey. Bulletin of the World Health Organization, 92: 204-213A.doi: http://dx.doi.org/10.2471/BLT.13.128371.Alam, M.K. 1992. Medical ethno-botany of the Marma tribe of Bangladesh. Economic Botany 46(3): 330-330.

Alam, M.K., Choudhury, J. and Hassan, M.A. 1996. Some folk formularies from Bangladesh. Bangladesh J. LifeSci. 8(1): 49-63.

Alexiades, M.N., (ed.) 1996. Selected Guidelines for Ethno botanical Research: A Field Manual. The NewYork Botanical Garden, New York.

Emily, S., Rahman M., Hossain M. J, Nahar N., Fazul M. A., Islam N., Sultana R., Akhtar S., Haider M. S., Islam M.S., Rahman M.W., Uddin M.Z., Mondal U.K. and Luby S.L. 2010. Fatal outbreak from consuming Xanthium strumarium seedlings during time of food scarcity in northeastern Bangladesh. PLoS ONE 5(3) |e9756.doi:10.1371/journal.pone.0009756.

Firedman, J., Yaniv, Z. Dafni, A. and Palewitch, D. 1986. A preliminary classification of healing potential plants, based on a rational analysis of an ethno pharmacological field survey among Bedouins in the Negev Desert, Israel. Journal of Ethno Pharmacology 16: 275-287.

Grover, J.K., Yadav, S., and Vats, V. 2002. Medicinal plants of India with anti-diabetic potential. J Ethnopharmacol, 81:81-100.

Haque, M., Uddin M.Z., Hassan M.A. and Saha M.L. 2017. Plants used for the treatment of diabetes inBrahmanbaria, Bangladesh. Clinical and Experimental homoepathy 4(3): 8-18.

Haque, T., Uddin M. Z., Saha M.L., Mazid M.A. and Hassan M.A. 2014. Propagation, antibacterial activity and phytochemical properties of Litsea glutinosa (Lour.) C. B. Robinson Dhaka Univ. J. Biol. Sci. 23(2): 165-171.

Hasani-Ranjbar, S., Larijani, B., and Abdollahi, M.A. 2009. A Systematic review of the potential herbal sources of future drugs effective in oxidant-related diseases. Inflamm Allergy Drug Targets 8: 2-10.

Hassan, M.A. and Khan, M.S. 1986. Ethnobotanical record of Bangladesh-1: Plants used for healing fractured bones. J. Asiatic Soc. Bangladesh. (Sci.). 12(1\&2): 33-39.

Hassan, M.A. and Khan, M.S. 1996. Ethnobotanical record of Bangladesh-2. Plants used for healing cuts and wounds. Bangladesh J. Plant Taxon. 3(2): 49-52.

Heinrich, M., Ankli, A., Frei, B. and Weimann, C. 1998. Medicinal plantsin Mexico: healers consensus and cultural importance. Social Science and Medicine 47: 1859-1871.

Hooker, J.D. 1872-1897. Flora of British India 1-7. First Indian Reprint 1973 Bishen Singh Mahendra Pal Singh, Dehra Dun, India.

Hyland, B.P.M. 1972. A technique for collecting botanical specimens in rain forest. Flora Malesiana Bulletin 26: $2038-2040$. 
Islam, M.R., Uddin, M.Z., Hassan, M.A. and Bhuiyan, M.N.I. 2010. Ethnobotanical survey of medicinal plants and their ethnic uses in Ramgarh Upazila of Khagrachari district, Bangladesh. Hamdard Medicus 53(3): 38-44.

Khan, M.S., Uddin M.Z. and Hassan M.A. 2002. Ethnobotanical survey in Rema-Kalenga wildlife sanctuary(Habiganj) in Bangladesh. Bangladesh J. Plant Taxon. 9(1): 51-60.

Mentreddy, S.R., Mohamed, A.I., Rimando, A.M., 2005. Medicinal plants with hypoglycemic/anti hyperglycemicproperties: a review. Proc Assoc Adv Ind Crop Conf. 20: 341-353

Mia, A. S. and Haque A. 1988. Ethnodiversity of medicinal plants used by Tripura community of Hazarikhil in Chittagong District of Bangladesh, Department of Botany, University of Chittagong, Chittagong 4331, Bangladesh, J. Taxon. Biodiv. Res. 5: 27-32.

Modak, M., Dixit, P., Londhe, J., Ghaskadbi, S., Paul, A., Devasagayam, T. 2007. Indian herbs and herbal drugs for the treatment of diabetes. J Clin Biochem Nutr. 40: 163-173.

Mukherjee, P.K., Maiti, K., Mukherjee, K. and Houghton, P.J., 2006. Leads from Indian medicinal plants with hypoglycemic potentials. J Ethnopharmacol. 106:1-28

Prain, D. 1903. Bengal Plants Vol. 1-2: 1-1013pp. First Indian Reprint 1963, Bishen Singh Mahendra Pal Singh Dehra Dun.

Roy, S., Uddin M.Z. Hassan M.A. and Rahman M.M. 2008. Medicobotanical report on the Chakma people of Bangladesh. Bangladesh J. Plant Taxon. 15(1): 67-72

Sajib, N.H. and Uddin S.B. 2013. Medico-Botanical Studies of Sandwip Island in Chittagong, Bangladesh. Bangladesh J. Plant Taxon. 20(1): 39-49.

Shethi, KJ and Uddin. MZ. 2018. Antioxydant properties of five commercially less valued vegetables from Bangladesh. Bangladesh J. Bot. 47(4): 953-959.

Siddiqui K.U., Islam, M.A., Ahmed, Z.U., Begum, Z.T.N., Hassan, M.A., Khondker, M., Rahman, M. M.,Kabir, S.M.H., Ahmad, M., Ahmed, A.T.A., Rahman, A.K.A. and Haque, E.U. (Eds), 2007. Encyclopedia of Flora and Fauna of Bangladesh, Vol. 11. Angiosperms; Monocotyledons. Asiatic Society of Bangladesh, Dhaka.

Uddin, M.Z., Khan M.S. and Hassan M.A. 2001. Ethnomedical Plant records of Kalenga forest range (Habiganj), Bangaldesh for Malaria, Jaundice, Diarrhoea and Dysentery. Bangladesh J. Plant Taxon. 8(1): 101-104.

Uddin, M.Z. and Hassan, M.A. 2004. Flora of Rema-Kalenga Wildlife Sanctuary. IUCN Bangladesh Country Office, Dhaka, Bangladesh, vi+120 pp.

Uddin, S.N., Hassan, M.A. and Rhaman M. 2004. Preliminary ethnomedical plant survey in Khagrachari district Bangladesh. Bangladesh J. Plant Taxon. 11(2): 39-48.

Uddin, M.Z., Hassan M.A. and Sultana M. 2006. Ethnobotanical survey of medicinal plants in Phulbari Upazila of Dinajpur district, Bangladesh. Bangladesh J. Plant Taxon. 12(1): 63-68.

Uddin, M.Z. and Roy S. 2007. Collection and Management of Selected Medicinal Plants in Rema Kalenga Wildlife Sanctuary.In: Making Conservation Work: Linking rural livelihoods and protected area management in Bangladesh edited by Fox, J. Bushley, B.R., Dutta S. and Quazi, S.A. 2007. Monograph of East-West Center, Hawaii, USA. pp. 66-83.

Uddin, M.Z., Hassan M.A., Rahman M.M. and Arefin M.K. 2012. Ethno-medico-botanical study in Lawachara National Park, Bangladesh. Bangladesh J. Bot. 41(1): 97-104.

Uddin, M.Z. and Hassan M.A. 2014. Determination of informant consensus factor of ethnomedicinal plants used in kalenga forest, Bangladesh. Bangladesh J. Plant Taxon. 21(1): 83-91.

Uddin, M.Z, Kibria M.G. and Hassan M.A. 2015. Study of ethnomedicinal plants used by the local people of feni district, Bangladesh. Journal of Asiatic society, Sci. 41(2): 203-223.

Uddin, M.S., Chakma J.J., Alam K.M.M. and Uddin S.B. 2015a. Ethno-medico Studies on the Uses of Plant in the Chakma Community of Khagrachari District, Bangladesh. J. Med. Plants Stud. 3(1): 10-15.

Uddin, M.S., Chowdhury V., Uddin S.B., Mazumder A.A.M. and Howlader M.S.A. 2015b. Ethnobotanical Survey of Medicinal Plants Used By the Lushai Community in Bandarban District, Bangladesh. J. Advanced Botany and Zoology, V 2I4. DOI: 10.15297/JABZ.V2I4.04 
Uddin, M.Z., Arefin M.K., Alam M.F., Kibria M.G., Podder S.L. and Hassan M.A. 2017. Consensus of the people's healthcare knowledge in the use of ethno-medicinal plants in and around lawachara national park. Journal of Asiatic Society 43(1): 101-123.

Uddin, MZ and Haque, T. 2018. Plants used for the beauty care in Dhaka, Bangladesh. Clinical and Experimental homeopathy 5(1): 29-35.

Uddin, MZ, Rifat, AB, Mitu, FY and Haque, T. 2019. Ethnomedicinal plants for prevention of cardiovascular diseases in Bangladesh. Bangladesh J. Plant Taxon. 26(1): 83-95.

Yusuf, S. 2006. Ethnobotanical investigation into the Mandi ethnic, Bangladesh J. Plant Taxon. 14(2): 129-145.

Yusuf, M. and Uddin J. 2006. Ethno-medico-botanical knowledge from Kaukhali proper and Betbunia of Rangamati District, Bangladesh J. Plant Taxon. 13(1): 55-61

Yusuf, M., M.A. Rahman, J.U. Choudhury and J. Begum, 2002. Indigenous knowledge about the use of Zingibers in Bangladesh. J. Econ. Taxon. Bot. 26(3): 566-570.

(Manuscript received on 3 May, 2019; revised on 10 November, 2019) 